\title{
Relativistic Models of Nonlinear Quantum Mechanics
}

\author{
T. W. B. Kibble \\ Blackett Laboratory, Imperial College, London SW7 2BZ, England
}

\begin{abstract}
I present and discuss a class of nonlinear quantum-theory models, based on simple relativistic field theories, in which the parameters depend on the state of the system via expectation values of local functions of the fields.
\end{abstract}

\section{Introduction}

The linearity of quantum mechanics, expressed in the "superposition principle" is anomalous. Linearity is a common feature of physical theories, but in all other known cases it is an approximation. The range over which linearity holds may be extensive, but is always limited: Maxwell's equations break down for very intense fields (when pair creation is important) and the linearity of space-time itself is a weak-field approximation.

Hitherto all the specific nonlinear generalizations of quantum theory that have been proposed have been nonrelativistic, or at best one-particle relativistic theories $[1,2]$. However, if there is a nonlinear theory underlying quantum mechanics, it seems likely that when the nonlinearity is important interparticle interaction, and particle creation and annihilation, are important too. Thus we should really be seeking a nonlinear generalization not of a one-particle Schrödinger equation but of a quantum field theory.

In this paper, I present a class of nonlinear quantum models based on simple field theories, and discuss their properties. It appears that these models, and some nonrelativistic ones, do not fit neatly into the formal schemes that have been set up to accommodate generalizations of quantum mechanics, such as the "convexity" formalism [1,3-7] based on the operational approach of Haag and Kastler [8]. I shall indicate some of the problems in this paper, and discuss a new formal structure in a later publication.

Quantum mechanics is among the most successful of physical theories. Many of its predictions, especially those of quantum electrodynamics, have been verified with unparalleled precision. So it may seem foolhardy to question its most basic tenet. On the other hand, despite its successes, the interpretation of quantum mechanics remains problematic [9]. There is still no generally accepted solution to the problem of "reduction of the wave packet". Moreover, there are grave difficulties confronting relativistic quantum theory, difficulties which have been 
circumvented but not eliminated by renormalization theory. Their resolution may well require a thorough reappraisal of the basic principles on which the theory is founded.

The superposition principle is hard to justify within an axiomatic framework, except on grounds of simplicity [10-14]. The basic difficulty is that the states of quantum mechanics are represented not by vectors but by rays in Hilbert space, or more generally by density operators. Although linearity itself is a simple concept, the conditions it imposes on the set of states--derivable from Gleason's theorem [15] - are far from simple. For this reason, a direct experimental test of the superposition principle is hard to devise. Bell's inequality [16] has been checked in several experiments [17-20] but it serves to exclude a class of hidden-variable theories [21] rather than to test the hypothesis that quantum mechanics is a linear approximation to some more general theory as has been suggested by de Broglie [22] and, in connection with the problem of conciousness, by Wigner [23].

In Section 2 I briefly recall some features of the simple nonrelativistic model based on the nonlinear Schrödinger equation, pointing out in particular the difficulty of finding a consistent measurement theory for it. Then in Section 3 I present the new generalized field-theoretic models, based on the idea that the parameters defining the theory, such as masses and coupling constants, may be state-dependent. Some solutions for a class of simple models are presented in Section 4, and the conclusions discussed in Section 5.

\section{Nonrelativistic Models and Measurement Theory}

Let us consider a nonrelativistic particle whose pure quantum states are described by solutions not of the linear Schrödinger equation but of some nonlinear generalization of it. If we want to preserve its locality properties, as well as its phase invariance, we are essentially restricted to adding terms proportional to $\psi$ multiplied by functions of $|\psi|^{2}$, perhaps with derivatives inserted. Galilean invariance imposes yet further restrictions. The simplest generalization satisfying these conditions is the well-known non-linear Schrödinger equation. If we include an external potential $V(x)$ it is

$$
i \frac{\partial \psi}{\partial t}=-\frac{1}{2 m} \nabla^{2} \psi+V \psi+\lambda|\psi|^{2} \psi
$$

where $\lambda$ is a parameter describing the strength of the nonlinear interaction. This equation has already been discussed in the context of non-linear quantum mechanics by Mielnik [1]. Other models of the same general type have been considered by Bialynicki-Birula and Mycielski [2].

The phase invariance leads directly to conservation of the norm:

$$
\int|\psi|^{2} d^{3} \mathbf{x}=\text { constant }
$$

so it is possible consistently to impose the normalization condition,

$$
\int|\psi|^{2} d^{3} \mathbf{x}=1
$$


and to interpret $|\psi|^{2}$ as the probability density for measurements of position. However, it should be noted that (2) is not merely a convenient conventional choice, as it is in ordinary quantum mechanics, because the equation (1) lacks the scaling invariance $\psi \mapsto \alpha \psi$.

The invariance under time translations leads to an exact conservation law for the "energy functional"

$$
\int\left\{\frac{1}{2 m}|\nabla \psi|^{2}+V|\psi|^{2}+\frac{1}{2} \lambda|\psi|^{4}\right\} d^{3} \mathbf{x}=\text { constant }
$$

but there is no obvious definition of an energy observable. When $V=0$, there is a corresponding exact conservation law for momentum.

If the pure states of a single nonrelativistic particle were described by normalized solution of (1), then clearly the ordinary linear Schrödinger equation would provide a good approximation so long as $\lambda|\psi|^{2}$ is small compared to the typical energies involved, that is to say except for states in which $|\psi|^{2}$ becomes very large at some point. Provided that $\lambda \ll e^{2} a_{0}^{2}$ (where $a_{0}$ is the Bohr radius), atomic energy levels would not be much affected.

However there are very serious problems attached to Equation (1). ${ }^{1}$ Let us consider its measurement theory. We have already agreed that $|\psi|^{2}$ can be interpreted as a probability density for measurements of position. But this is only half of what we need. We may also ask what is the effect of the measurement on the state of the particle. Suppose that the apparatus consists of a counter of some kind which either clicks or does not click depending on whether the particle is or is not inside its volume $\Omega$ at a given time. (It may be imperfect, of course, and sometimes give an incorrect result, but this does not affect the argument in any essential way). Then we may ask: if the state of the particle before the measurement is described by $\psi$, and if on this occasion the counter clicks, then what is the state $\psi^{\prime}$ after the measurement?

In ordinary quantum mechanics the answer is straightforward, at least for an "ideal" measurement. We first apply the projection operator defined by $\Omega$ (i.e. set $\psi$ equal to zero outside $\Omega$ while leaving it unchanged inside) and then rescale to recover a normalized wave function $\psi$ '. This is the "reduction of the wave packet".

The difficulty in our case is that rescaling is no longer a trivial operation, so it is far from obvious that this answer can be taken over unchanged.

There are several possibilities:

1. The same reduction of the wave packet may apply. This would have the rather peculiar effect that because of the rescaling the time dependence of the wave function would suddenly change, because the nonlinear term would become more important. For example, if the particle is found not to be in $\Omega$, the time dependence of the wave function even far away from $\Omega$ would change suddenly. In a full treatment of the problem we should of course include both particle and apparatus within our system and set up some kind of interaction between the two. It is very

1 After completing this work, it came to my attention that a very similar argument has recently been put forward by Haag and Bannier [28] 
difficult to think of any kind of interaction that would have the effect envisaged.

2. Possibly our choice of the set of pure states is too restrictive and we should allow states with norm less than one. In that case however each measurement would apparently reduce the norm, so it is not at all clear how states of unit norm could be created in the first place.

3. Conceivably the result of the measurement might not be a pure state at all. However, this does not help, because we can then ask what are the pure states of which it is a mixture, and essentially the same problems apply to them. Moreover it seems implausible that a simple measurement on a pure state should lead to a statistical mixture (unless of course the result is unknown).

4. Since none of these possibilites seems very attractive we could try to modify the equation so that it has a scaling invariance, i.e. to make it homogeneous of degree one, though nonlinear. For example, we could introduce a nonlinear term of the form $\lambda|\nabla \psi|^{2} \psi /|\psi|^{2}$. However such a term spoils the Galilean invariance of the theory, and moreover there are potential singularities when $\psi=0$. A much better solution is the logarithmic model of Bialynicki-Birula and Mycieski [2] which is not quite homogeneous of degree one, but in which rescaling corresponds only to an overall phase change. In that model one could take over unchanged the usual "reduction of the wave packet".

5. Possibly the right conclusion is that we should abandon the idea of a nonlinear one-particle model. Even in a non-relativistic context, it is hard to believe in noninteracting particles in a regime where the individual particles are described by nonlinear equations. It appears much more plausible that when nonlinearity is important interaction between particles is also important. Indeed if the particles are indistinguishable this seems almost inescapable.

Let us proceed therefore to try to find a relativistic many-particle model.

\section{Field-Theoretic Models}

In any interacting field theory the equations of motion for the fields are nonlinear, but this is of course a quite different kind of nonlinearity from the one we are discussing, which is a nonlinearity in the state-vector. In looking for nonlinear generalizations of field theories it is best therefore to start with a formalism in which the state-vector appears explicitly, i.e. to work in the Schrödinger rather than the Heisenberg picture.

In the nonrelativistic model considered in the previous section, the states of the system at a given time are represented by functions of exactly the same class as in the conventional linear theory. The nonlinearity enters only through the timedependence, which is now described by a nonlinear equation. Let us now consider relativistic models constructed in the same way. This means that the states at a given time may be represented as usual by normalized vectors $\langle\Psi, t\rangle$ in a complex Hilbert space $\mathscr{H}$, but their time-dependence is now given by a generalized Schrödinger equation of the form

$$
i \frac{\partial}{\partial t}|\Psi, t\rangle=H_{\Psi}|\Psi, t\rangle
$$


which is nonlinear by virtue of the fact that the Hamiltonian operator $H_{\Psi}$ depends explicitly on the state $\Psi$.

In order to preserve the locality of the theory, we must require that

$$
H_{\Psi}=\int d^{3} \mathbf{x} \mathscr{H}_{\Psi}(\mathbf{x}),
$$

where $\mathscr{H}_{\Psi}(\mathbf{x})$ is a local function of the field operators and their derivatives, and depends on local characteristics of the state $\Psi$. Consider for simplicity a theory of a single scalar field $\phi$ which with its canonical conjugate $\pi$ satisfies the canonical commutation relations

$$
[\phi(\mathbf{x}, t), \pi(\mathbf{y}, t)]=i \delta_{3}(\mathbf{x}-\mathbf{y}) .
$$

Then a simple way of implementing the nonlinearity is to make the parameters of the theory, like the mass and coupling constant, dependent on the state of the system via the expectation values of some local operators. For example, as a generalization of the usual $\phi^{4}$ theory, we may take

$$
\mathscr{H}_{\Psi}(\mathbf{x})=\frac{1}{2} \pi^{2}(\mathbf{x})+\frac{1}{2}[\nabla \phi(\mathbf{x})]^{2}+\mathscr{V}_{\psi}(\phi(\mathbf{x})),
$$

where $\mathscr{V}_{\Psi}$ is a quartic polynomial

$$
\mathscr{V}_{\Psi}(\phi)=\langle f(\phi)\rangle_{\Psi}+\frac{1}{2}\langle h(\phi)\rangle_{\Psi} \phi^{2}+\frac{1}{24}\langle g(\phi)\rangle_{\Psi} \phi^{4} .
$$

Here $f, h$ and $g$ are local functions of the field $\phi(\mathbf{x})$, and the expectation values are defined in the usual way; for instance,

$$
\langle f(\phi)\rangle_{\Psi}=\frac{\langle\Psi|f(\phi)| \Psi\rangle}{\langle\Psi \mid \Psi\rangle} .
$$

Notice that because of the explicit denominator in this definition, the Schrödinger Equation (3) is invariant under the rescaling $|\Psi\rangle \mapsto \lambda|\Psi\rangle$. It is consistent to impose the normalization condition

$$
\langle\Psi \mid \Psi\rangle=1
$$

which is then preserved in time. Thus the problem associated with "reduction of the wave packet", discussed in the preceding section, does not arise for this model. On the other hand the scalar product $\langle\Psi \mid \Phi\rangle$ of two different states is not time-independent in general.

The models described above have many desirable features, as we shall see: they are Lorentz-invariant and local, and reduce to standard linear quantum field theories for states that are sufficiently "diffuse".

Lorentz-invariance is easy to establish, though of course the realization of Lorentz transformations, like that of time translations, is nonlinear. The essential point is that although the Hamiltonian density $\mathscr{H}$ depends on $\Psi$ it satisfies (in the usual formal sense) a commutation relation independent of $\Psi$, namely

$$
\left[\mathscr{H}_{\Psi}(\mathbf{x}), \mathscr{H}_{\Psi}(\mathbf{y})\right]=i \nabla \cdot\left\{\delta_{3}(\mathbf{x}-\mathbf{y}) \pi(\mathbf{x}) \nabla \phi(\mathbf{x})\right\}-(\mathbf{x} \leftrightarrow \mathbf{y}) .
$$

This is enough [24] to establish that the generators obey the Lie algebra of the Lorentz group. (Invariance under translations and spatial rotations is of course trivial). 
The theory is local in essentially the usual sense. We have hitherto taken the field operator $\phi$ as a basic, undefined entity, but it can be defined operationally in terms of the response of the system to a small external perturbation. Then in general the response to a finite perturbation should be represented by a unitary operator function of $\phi$, possibly state-dependent. If the perturbation is confined to a finite volume, then it does not alter the expectation values of functions of $\phi$ outside this volume, and consequently does not affect the time evolution of the state in relatively spacelike regions.

Since $f, h$ and $g$ are local functions of $\phi(\mathbf{x})$, it is clear that their expectation values will be significantly different from those in the vacuum only for states in which (in a Fock-space picture) some of the particle wave functions are reasonably large near $\mathbf{x}$. For states which are sufficiently diffuse, in the sense that no particle wave function is concentrated in any particular region, the functions $f, h$ and $g$ may be replaced by their vacuum expectation values. Then we recover the conventional $\phi^{4}$ theory. For example, suppose that the three functions depend linearly on $\phi^{2}$. Then a state $|\Psi\rangle$ may be regarded as near the vacuum if $\left\langle\phi^{2}\right\rangle_{\Psi} \ll m^{2}$, where $m$ is some characteristic mass, depending on the parameters of the model. For such nearly vacuous states, the theory reduces to the usual linear theory.

\section{Solutions of Field-Theoretic Models}

There are of course no explicit solutions known of the fully interacting $\phi^{4}$ theory. However we can gain some insight into the effects of the nonlinearity by studying models with $g=0$. Although these are in a sense generalized free-field models, they do not represent noninteracting particles because the nonlinearity itself induces interaction.

To be specific let us suppose that both $f$ and $h$ are linear functions of $\phi^{2}$. The expectation value of $\phi^{2}$ is of course infinite in any state, because of the usual divergence in the limit as $\mathbf{y} \rightarrow \mathbf{x}$ in $\langle\phi(\mathbf{x}) \phi(\mathbf{y})\rangle$. To render it finite we must subtract out the vacuum expectation value. Let us therefore define

$$
: \phi^{2}:=\phi^{2}-\left\langle\phi^{2}\right\rangle_{0},
$$

where the subscript zero denotes the expectation value in a specific state $\left|\Psi_{0}\right\rangle$ to be determined below, and take

$$
f=\alpha+\beta: \phi^{2}:, \quad h=m^{2}+\lambda: \phi^{2}: .
$$

The value of the constant $\alpha$ may be adjusted to make the vacuum energy density zero; $m$ is the mass in near-vacuum states; $\beta$ and $\lambda$ are new parameters describing the nonlinearity. For the present, we assume that $\lambda>0$.

It is easy to find a class of stationary solutions of the Schrödinger equation. Let us enclose the system in a box of volume $V$, impose periodic boundary conditions, and look for states that are unchanged up to a phase by time or space translations. Because of the translation invariance $\left\langle: \phi^{2}:\right\rangle_{\Psi}$ is a constant in any such state $\Psi$. Let us pick a value for this constant, to be fixed later by a consistency condition. Then the Hamiltonian density,

$$
\mathscr{H}_{\Psi}=\frac{1}{2} \pi^{2}+\frac{1}{2}(\nabla \phi)^{2}+\frac{1}{2}\langle h\rangle_{\Psi} \phi^{2}+\langle f\rangle_{\Psi},
$$


is that of a system of free particles of squared mass $\langle h\rangle_{\Psi}$. Any stationary state can be labelled by the occupation numbers $n_{k}$ for the various modes. The energy eigenvalue (not the value of the energy functional) is

$$
E=\sum_{\mathrm{k}}\left(n_{\mathrm{k}}+\frac{1}{2}\right) \omega_{\mathrm{k}}+\langle f\rangle V
$$

where $\omega_{\mathbf{k}}$ is given by

$$
\omega_{\mathbf{k}}^{2}=\mathbf{k}^{2}+\langle h\rangle=\mathbf{k}^{2}+m^{2}+\lambda\left\langle: \phi^{2}:\right\rangle .
$$

To ensure that $E=0$ for the vacuum state $\left|\Psi_{0}\right\rangle$, with all $n_{\mathrm{k}}=0$, we must take

$$
V \alpha=-\sum_{\mathrm{k}} \frac{1}{2} \omega_{\mathrm{k}}^{0}
$$

with

$$
\omega_{\mathbf{k}}^{0}=\left(\mathbf{k}^{2}+m^{2}\right)^{1 / 2}
$$

The value of $\left\langle: \phi^{2}:\right\rangle$ is obtained from the consistency condition

$$
\left\langle: \phi^{2}:\right\rangle=\frac{1}{V} \sum_{\mathrm{k}}\left(\frac{n_{\mathrm{k}}+\frac{1}{2}}{\omega_{\mathrm{k}}}-\frac{\frac{1}{2}}{\omega_{\mathrm{k}}^{0}}\right),
$$

where the second term inside the sum is determined by the requirement that $\left\langle: \phi^{2}:\right\rangle_{0}=0$.

Thus, for any chosen set of integers $n_{k}$, the value of $\left\langle: \phi^{2}:\right\rangle$ may be found by solving the implicit equation

$$
\left\langle: \phi^{2}:\right\rangle=\frac{1}{V} \sum_{\mathrm{k}}\left(\frac{n_{\mathrm{k}}+\frac{1}{2}}{\left[\mathbf{k}^{2}+m^{2}+\lambda\left\langle: \phi^{2}:\right\rangle\right]^{1 / 2}}-\frac{\frac{1}{2}}{\left[\mathbf{k}^{2}+m^{2}\right]^{1 / 2}}\right) .
$$

Since the right side is (for $\lambda>0$ ) a monotonically decreasing function, positive at $\left\langle: \phi^{2}:\right\rangle=0$, there is always a unique solution. Then the frequencies $\omega_{\mathbf{k}}$ are given by (13), and the energy $E$ by

$$
E=\sum_{\mathrm{k}}\left[\left(n+\frac{1}{2}\right) \omega_{\mathrm{k}}-\frac{1}{2} \omega_{\mathrm{k}}^{0}\right]+\beta V\left\langle: \phi^{2}:\right\rangle .
$$

In the special case where $\lambda=0$, the model becomes essentially trivial and can be solved completely. Then $\omega_{\mathbf{k}}=\omega_{\mathbf{k}}^{0}$ for all states, and the energies of stationary states are given by

$$
E=\sum_{\mathrm{k}} n_{\mathrm{k}}\left(\omega_{\mathrm{k}}+\frac{\beta}{\omega_{\mathrm{k}}}\right) .
$$

However, in this case the nonlinear term in the Hamiltonion is merely a c-number, albeit state-dependent. Thus if $|\Psi, t\rangle_{0}$ denotes any solution of the linear Schrödinger equation obtained by setting $\beta=0$, a corresponding solution of the nonlinear equation is

$$
|\Psi, t\rangle=|\Psi, t\rangle_{0} e^{-i \sigma(t)}
$$

where the phase factor $\sigma$ is the solution of the differential equation 


$$
\frac{d \sigma}{d t}=\beta \int d^{3} \mathbf{x}_{0}\left\langle\Psi, t\left|: \phi^{2}(\mathbf{x}):\right| \Psi, t\right\rangle_{0}
$$

with $\sigma(0)=0$.

If we assume that the only observables in the theory are functions of $\phi$, it follows that the introduction of a time-dependent phase factor is physically irrelevant. Thus $\beta$ has no physical significance, and without essential loss we may set it equal to zero. Returning to the case $\lambda \neq 0$, we find that the energy is then given by

$$
E=\sum_{\mathrm{k}}\left[\left(n_{\mathrm{k}}+\frac{1}{2}\right) \omega_{\mathrm{k}}-\frac{1}{2} \omega_{\mathrm{k}}^{0}\right]
$$

In the limit $V \rightarrow \infty$ we find from (15) that if only finitely many occupation numbers $n_{k}$ are different from zero then $\left\langle: \phi^{2}:\right\rangle \rightarrow 0$. This is to be expected, because the finite number of particles is spread out over infinite volume, so that the state becomes infinitely diffuse. Nonlinear effects will be important in this model only for wave-packet states with small uncertainty in position.

Qualitatively it is not hard to see what these effects would be. The nonlinear term effectively alters the mass of a propagating particle when it is in a region of large $\left\langle: \phi^{2}:\right\rangle$. Thus there will be scattering even without the presence of a $g \phi^{4}$ term. Approximate plane-wave states will not scatter, but sharply concentrated wave-packet states will do so. Qualitatively, the effect will be a mutual refraction of particles, and even a self-refraction, rather similar to the well-known effects in nonlinear optics [25]. I shall not attempt to discuss the details here. In any case they must depend strongly on the nature of the function $h(\phi)$. Here it has been chosen to be quadratic only for reasons of simplicity; other choices may have to be considered.

We have found a large class of stationary solutions; indeed in the case $\lambda=0$, it includes all stationary solutions. When $\lambda>0$ there are probably no other solutions, because the nonlinear term yields effectively a repulsive potential. However, for sufficiently large negative values of the dimensionless parameter $\lambda$, there may well be other "bound-state" stationary solutions, in which $\left\langle: \phi^{2}:\right\rangle$ would have a spatial dependence. There would of course necessarily be a degenerate family of solutions related by translational invariance, but whereas in a linear theory one could always superimpose these solutions to yield a translationally-invariant solution, this is not possible in our nonlinear model. We might well have quantum solitons, like the "gaussons" obtained by Bialynicki-Birula and Mycielski [2]. However such solutions have not yet been found.

\section{Discussion}

In the last two sections, we have discussed a simple nonlinear generalization of the scalar field theory with $\phi^{4}$ interaction. It is clear that similar generalizations could be written down of other field theories such as quantum electrodynamics. If the nonlinearity parameters are chosen small enough, then for a wide range of phenomena the ordinary linear theory will apply. It should not be difficult to preserve the successful features of conventional field theory. Whether it is possible 
at the same time to eliminate some of its less desirable features such as divergences is not immediately clear. To answer this question we have first to set up a perturbation theory. This is problematic because we no longer have a complete orthonormal set of unperturbed energy eigenstates in terms of which to expand.

There are perhaps two areas where models of this kind might find application. One is in connection with the problem of "confinement" in the theory of hadronic structure, along the lines of the model proposed by Lee and Wick [26], where a field has different expectation values inside and outside a "bag", giving different effective particle masses in the two regions. The other is to quantum gravity. It has often been remarked (for example in [1]) that there is an apparent mismatch between the essentially nonlinear classical theory of general relativity and the linearity of quantum mechanics. Indeed Penrose has recently suggested [27] using a nonlinear description even for a single graviton. It seems plausible that at the very short distances (of the order of the Planck length) where quantum fluctuations of the space-time metric become significant, the manifold of quantum states can no longer be treated as flat.

It would also be possible within this framework to discuss the effect of a quantized matter field on an unquantized gravitational background, via the equation

$$
G_{\mu \nu}=8 \pi \kappa\left\langle T_{\mu \nu}\right\rangle .
$$

The fact that this equation leads to a nonlinear quantum evolution equation is no bar.

It would be natural to try to fit the models constructed here into the axiomatic framework for generalized quantum mechanics formulated by Mielnik [1] on the basis of the "convexity" formalism. Indeed, this will be discussed in detail in a later publication. However, there is a basic difficulty, common to many nonrelativistic models too, though not always recognised. There is a sense in which a nonlinear model of this kind is more akin to classical than to quantum mechanics. In quantum mechanics, there is a well-defined metric on the manifold of pure states, so that one can sensibly talk about one state being near to another. Classically on the other hand any two pure states can be distinguished with certainty by some measurement, and there is no notion of nearness of states. One can of course define a metric in phase space, but in general two states that are initially close will move arbitrarily far apart over the course of time. But the same is almost certainly true of our nonlinear models. The states at any given time are described by vectors in the Hilbert space, in which there is of course a metric. However apart from very exceptional cases the time-development described by a nonlinear equation will carry initially neighbouring states far apart. Restricted only by the few exact conservation laws, the states will move over the available region of state space in a more or less ergodic fashion. It follows from this that if one is willing to wait long enough any two pure states can be distinguished with certainty just as they can in classical mechanics. ${ }^{2}$ Nevertheless, in a real physical sense the

\footnotetext{
2 Essentially the same point has recently been made by Haag and Bannier [28], and also by Mielnik in an unpublished note
} 
models presented here are surely quantal rather than classical. The modification in the general axiomatic framework needed to accommodate them will be discussed in a later publication.

Acknowledgement. I am indebted to Dr. C. J. Isham for valuable suggestions.

\section{References}

1. Mielnik, B. : Commun. math. Phys. 37, 221 (1974)

2. Bialynicki-Birula, I., Mycielski, J. : Ann Phys. 100, 62 (1976)

3. Ludwig, G. : Deutung der Begriffs "physikalische Theorie" und axiomatische Grundlegung der Hilbertraumstruktur der Quantenmechanik durch Hauptsätze der Messens. In : Lecture notes in physics, Vol. 4. Berlin, Heidelberg, New York: Springer 1970

4. Davis, E. B., Lewis, J. T. : Commun. math. Phys. 17, 239 (1970)

5. Gunson, J. : Commun. math. Phys. 6, 262 (1967)

6. Gudder, S. P. : Four approaches to axiomatic quantum mechanics. In: The uncertainty principle and foundations of quantum mechanics (eds. W. C. Price, S. S. Chissick), p. 247. London: Wiley 1970

7. Edwards, C. M. : Commun. math. Phys. 16, 207 (1970)

8. Haag, R., Kastler, D. : J. Math. Phys. 5, 848 (1964)

9. Bastin, T. (ed.): Quantum theory and beyond. Cambridge: Cambridge University Press 1971

10. Varadarajan, V. C. : Geometry of quantum mechanics, Vol. I. Princeton, NJ : Van Nostrand 1968

11. Mackey, G. W. : Mathematical foundations of quantum theory. New York : Benjamin 1963

12. Jauch, J. M. : Foundations of quantum mechanics. Reading, Mass. : Addison-Wesley 1968

13. Piron, C. : Foundations of quantum physics. New York, Amsterdam: Benjamin 1976

14. Zabey, P. C. : Found. Phys. 5, 323 (1975)

15. Gleason, A. M. : J. Math. Mech. 6, 885 (1957)

16. Bell, J. S. : Rev. Mod. Phys. 38, 447 (1966)

17. Freedman, S. J., Clauser, J. F. : Phys. Rev. Letters 28, 938 (1972)

18. Kasday, L. R., Ullman, J., Wu, C. S. : Bull. Am. Phys. Soc. 15, 586 (1971)

19. Faraci, G., Gutkowski, D., Notarrigo, S., Pennisi, A. R. : Lett. Nuovo Cimento 9, 607 (1974)

20. Lamehi-Rachti, M., Mittig, W. : Phys. Rev. D14, 2543 (1976)

21. Belinfante, F. J. : A survey of hidden variables theories. Oxford: Pergamon Press 1973

22. de Broglie, L. : Nonlinear wave mechanics. Amsterdam: Elsevier 1960

23. Wigner, E. P. : Remarks on the mind-body question. In: The scientist speculates (ed. I.T. Good). London: Heinemann 1961

24. Schwinger, J. : Phys. Rev. 130, 406 (1963)

25. Kibble, T. W. B. : Phys. Rev. 150, 1060 (1966)

26. Lee, T. D., Wick, G. C. : Phys. Rev. D9, 2291 (1974)

27. Penrose, R. : Gen. Rel. Grav. 7, 171 (1976); 7, 31 (1976)

28. Haag, R., Bannier, U. : Commun. math. Phys. 60, 1 (1978)

Communicated by R. Haag

Received June 1, 1978 